Journal of Engineering and Applied Sciences 14 (Special Issue 7): 10131-10136, 2019

ISSN: 1816-949X

(C) Medwell Journals, 2019

\title{
Effect of the Magnetic Field Interacted with Two Aspergillus Species in Degradation of Some Chemical Pesticides
}

\author{
${ }^{1}$ Salwan Abd Alzahra Jabbar Allwbawi, ${ }^{2}$ Sabah Lateef Alwan, ${ }^{3}$ Nihad. H Mutlag, \\ ${ }^{4}$ Adeeb Kitab Abid Zaid Alshafiee and ${ }^{5}$ Hussein Rasim Sabboree \\ 1,2,4,5 Department of Plant Protection, Faculty of Agriculture, University of Kufa, Najaf, Iraq \\ ${ }^{3}$ Department of Ecology, Faculty of Science, University of Kufa, Najaf, Iraq
}

\begin{abstract}
The aim of this study was to investigate the degradation of chemical compounds of five pesticides (Bayfidan EC, Bulldock EC, Oberon SC, Galaxy SL and Trayf EC) using the magnetic field interacted with two fungal species Aspergillus falavus and A. terres. Pesticides in the concentrated form were exposed to a magnetic field (0) for a period of $720 \mathrm{~h}$ and were used based on the recommended dose in two different culture media (PDA and PDB) to test the biodegradation level by the two fungi. The insecticide Bulldock was more toxic after exposure to the magnetic field and resulted in more inhibition rate on $A$. flavus than nonmagnetized. Exposure to magnetic field did not differ from non-exposure in terms of $A$. terres growth on both Bulldock and Oberon culture media. The biodegradation of Trayf was slightly affected by magnetic field exposure while galaxy was not affected. Bayfidan degradation was affected by the magnetic field. This magnetized fungicide was more toxic to A. terres, causing an inhibition rate of 54.33\%.
\end{abstract}

Key words: Investigate the degradation, chemical compounds, inhibition, non-magnetized, stimulation, PDB

\section{INTRODUCTION}

In 2007, global sales of pesticides amounted to more than $\$ 30$ billion indicating that pesticides are still the most usable method in pest control although, the prior knowledge of the risks and damage caused by pesticides to the environment and public health. Alternative methods of pesticides in pest control such as biological control, agricultural practices, physical and genetic methods have led to the development of IPM programs and pest management systems which are striving to promote environmental means in pest control. To achieve food security, the world has begun of using modern and safe techniques to increase production because the continued use of pesticides to increase agricultural production has become a major harm to human health and serious environmental risk (Al-Malah and Al-Jubori, 2013). It is therefore, necessary to look for a strategy that will mitigate environmental decline while maintaining the agricultural production at satisfactory level quantitatively and quantitatively (Al-Adil and Khalid Mohamed, 2006; Dewan et al., 1994). Researchers have increased the interest of using magnetized water in adapting the properties of brackish and fresh water and their use for agricultural purposes. The magnetization process is accompanied by a range of changes in the chemical and physical properties of water including increasing dissolved oxygen, reduction of surface tension, increased solubility of solids and electrical conductivity, soil nutrients availability, improve of the cell membrane permeability and low viscosity compared with normal water. These changes in magnetized water will increase plant absorption leading to acceleration of vital processes and positively affects plant growth and development (Toledo et al., 2008).

Magnetized water was also used for pesticides dilution and to study its effect on the technical specifications of the pesticides. Name year (Gorski and Wachowiak, 2004) found that dilution of Karate 2.5 EC and Winglofos 550 EC. Using magnetized water resulted in significantly effective control of the two-point red dot mite Tetranychus urticae Koch and the rice weevil Sitophilus oryzae L. Al-Mallah et al. (2012) reported that magnetized water resulted in an increase in the thickness of the separation layer of the pesticide emulsion to $10.75 \mathrm{~mm}$ for the Flash when stored at $55^{\circ} \mathrm{C}$ for three months followed by Cyrin with an average separation layer of $6.4 \mathrm{~mm}$ compared to $0.2 \mathrm{~mm}$ of the control. Using magnetized water in degradation of chemicals including chemical pesticides would be an favorable, clean and environmental friendly method to reduce pesticides residues hazards. This study, therefor was conducted to investigate the possible use of magnetic field and magnetized water to reduce environmental pollution and to control pant pathogens.

\section{MATERIALS AND METHODS}

Isolation and diagnosis of pesticides degrading fungi from soil and organic fertilizers: Agriculture field soil and two organic fertilizers (rice and wheat compost from 
Organic fertilizer center/Kufa) were used as well as five different pesticides including the fungicide Bayfidan EC, insecticides Bulldock EC and Oberon SC and herbicides Galaxy SL and Trayf EC at their commercial concentrations (without dilution). The soil and organic fertilizers were evenly distributed $300 \mathrm{~mL}$ plastic pots and the pesticides degrading fungi were isolated by two methods.

First, pots with soil or compost were saturated with the commercial concentration of each pesticide individually with three replicates each and stored in the laboratory condition. Three months later, the fungi were isolated from each pot using the dilution method (Salwan, 2015).

The second method was to immerse sterilized sticks (tooth picks) in the concentrated pesticides for each pesticide for $48 \mathrm{~h}$. Three sticks were then embedded in each the pot of each type of potting soil mix with three replicates of each treatments. The pots were also stored in the laboratory condition for 3 months. Later, the sticks were pulled off and cultured in $9 \mathrm{~cm}$ Petri dishes with PDA to which the chloramphenicol was added at $250 \mathrm{mg} / \mathrm{L}$. The dishes were incubated at $25 \pm 2^{\circ} \mathrm{C}$. After 3 days, the percentage of occurrence and frequency of each presented fungus was calculated (Dewan et al., 1994). Present fungi were diagnosed in the graduate fungi laboratory in the Faculty of Agriculture, University of Kufa (Salwan, 2015). Since, the fungi Aspergillus flavus and A. terres were the most prevalent (occurrence frequency) fungi, they were used in this study experiments.

Pathogenicity test: Fungi Aspergillus flavus and A. terres were tested for their pathogenicity on radish seeds in a Water Agar medium (WA). The $9 \mathrm{~cm}$ petri dishes were filled with WA and inoculated (cultured) with either Aspergillus flavus isolate \#2 or A. terres isolate \#4 and incubated at 25>. About 3 days after, the dishes were planted with 20 radish deeds each with three replicates for each fungus. Percentages of seed germination, seed rot, infected seeds and healthy seeds were calculated 7 days post planting (Carling and Leiner, 1986).

Magnetic field effect on fungi (A. terres and A. flavus) growth in PDA poisoned with pesticide (Bayfidan EC, Bulldock EC ,Oberon SC, Galaxy SL and Trayf EC) medium: Pesticides (Bayfidan, Bulldock, Oberon, Galaxy and Trayf) were placed in $25 \mathrm{~mL}$ tubes and exposed to the magnetic field (24 m M Tesla) for a period of 0 or $72 \mathrm{~h}$ (Fig. 1). About $15 \mathrm{~mL}$ of each pesticide was randomly placed in a metal container and magnetized on four sides. The control treatment was placed in a similar container but without magnets. After each magnetizing period, the recommended dose of each pesticide was taken and added to sterilized PDA medium and poured in Petri dishes. The dishes were then inoculated in the center with $0.5 \mathrm{~cm}$ disk of A. terres or A. flavus cut from 7 days pure culture with three replicates each and incubated at $25 \pm 2^{\circ} \mathrm{C}$. Percent inhibition and/or stimulation was calculated according to Al-Malah et al. (1993).

Magnetic field effect on fungi (A. terres and A. flavus) growth in PDB liquid medium poisoned with pesticide (Bayfidan, Bulldock, Oberon, Galaxy and Trayf): Potato Dextrose Broth liquid medium (PDB) was used in this test. The PDB medium was sterilized and distributed in $250 \mathrm{~mL}$ flasks with $100 \mathrm{~mL}$ each. The flasks were

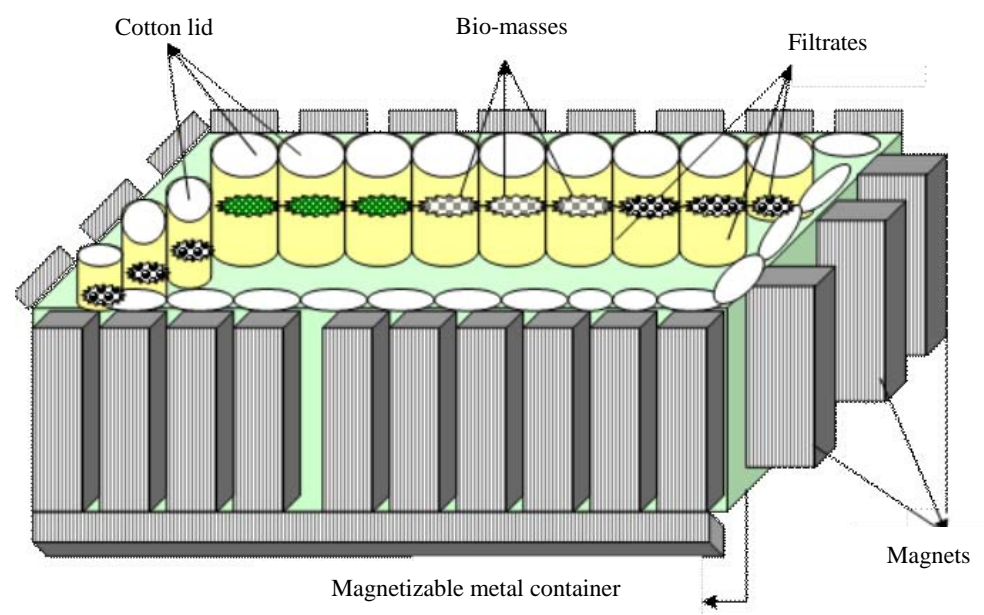

Fig. 1: A method to expose the bio-filtrates to the magnetic field 
inoculated with three disks of A. terres or A. flavus and the in the pesticide (Bayfidan, Bulldock, Oberon, Galaxy and Trayf) was added to the medium. The treatments were PDB with or without exposure to magnetic field and PDB poisoned with a pesticide (Bayfidan, Bulldock, Oberon, Galaxy and Trayf) with or without exposure to magnetic field. All treatments were replicated three times for each fungus and each pesticide. Same number of flasks were not poisoned (no pesticide). All experimental flasks were incubated at $25 \pm 2^{\circ} \mathrm{C}$ for 30 days with continuous exposure to the magnetic field (48 m M Tesla) and shaking every 2-3 days for even distribution. The biomass then was extracted with sterile forceps and placed on paper towels to remove the free water. Fresh weights were recorded and biomasses were placed in oven to dry at $70^{\circ} \mathrm{C}$ for $48 \mathrm{~h}$ and the final dry weight was recorded. Percent inhibition and stimulation were calculated as formerly mentioned (Salwan, 2015).

Experimental design and data analysis: Laboratory experiments were factorial based on CompleteRandomized-Design (CRD) with tree replicates. Data were analyzed and Analysis of Variance (ANOVA) was performed. Means were compared with the Least-Significant-Difference (LSD) whenever appropriate at $(\mathrm{p} \leq 0.05)$ (Gomez and Gomez, 1984).

\section{RESULTS AND DISCUSSION}

Pathogenicity tests of studied fungi: The results in Fig. 2 showed that the highest percentage of germination (100\%) was in the control treatment and the lowest (65\%)

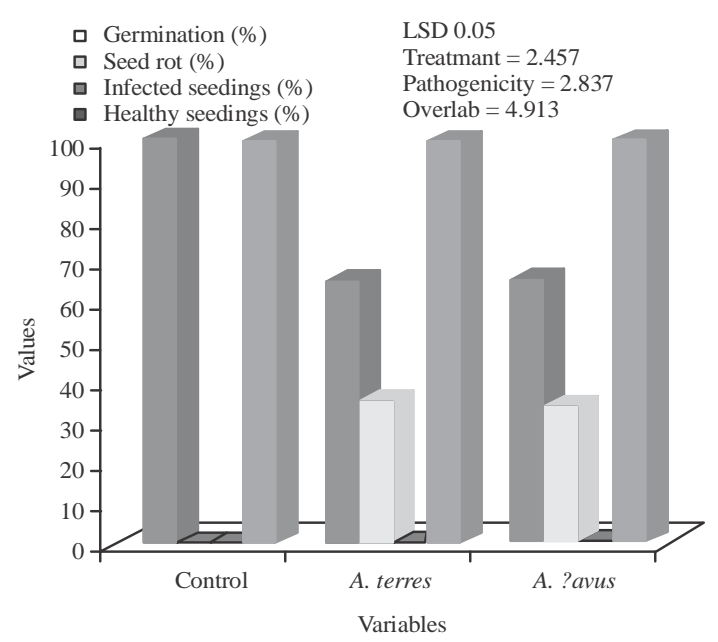

Fig. 2: Pathogenicity test of A. terres and A. flavus and their effect on radish seed germination, seed rot and seedlings health seven days after planting on WA in lab condition. Values are means of three replicates. Within treatment, bars with different letter are significantly $(\mathrm{p} \leq 0.05)$ different was in the treatment of A. terres in which significantly the highest percentage of rotting seeds (35\%) was recorded compared to no seed rot in the control treatment. All treatments did not affect seedlings health, seedlings of all treatments were healthy.

The reason for the high germination rate of seeds with both fungi is that these fungi are non-pathogenic and their secretions are non-toxic and did not affect the germination of seeds (Dewan et al., 1994). The increase in germination rate of radish seeds may be also due to these fungi secrete some substances that helps in seed outer shell degradation and accelerating seed germination. These substances may be enzymes such as cellulase or substances in the form of growth promoting such as Indol Acetic Acid (IAA) (Harman, 2000) or 2-carboxymethyl-3-n-hexyl malic acid (Mondal et al., 2000).

The effect of the magnetic field and fungi (A. terres and A. flavus) presence interaction on degradation of five pesticides (Bayfidan, Bulldock, Oberon, Galaxy and Trayf)

The insecticides Bulldock and Oberon: The growth of the two fungi A. flavus and A. terres was affected by different type of insecticide and the exposure to magnetic field (Table 1). Compared to the radial growth of both fungi in the control, the highest percentage of inhibition was found to be in the treatment of A. flavus and the lowest was in A. terres treatment, 13.39 and $0.00 \%$, respectively. The treatment of insecticide Bulldock with exposure to magnetic field resulted in the highest percentage of growth inhibition (12.09\%) compared to the lowest inhibition rate $(0.00 \%)$ from the control. For interaction treatment effect, the highest percentage of inhibition (24.18\%) was found in the magnetized Bulldock interacted with the A. flavus. Regardless of exposure to magnetic field, the radial growth of A. terres was not effected by Bulldock nor by Oberon (Table 1).

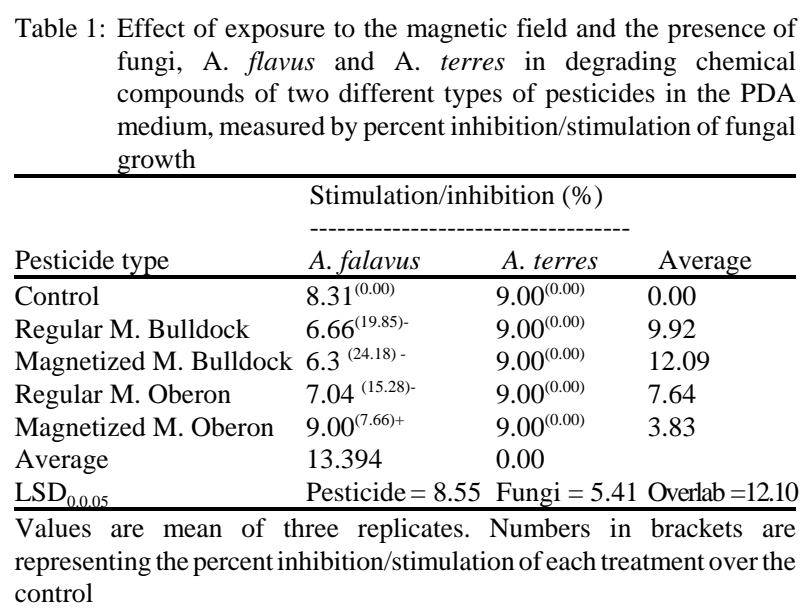


Table 2: Effect of exposure to the magnetic field and the presence of fungi, A. flavus and A. terres in degrading chemical compounds of two different types of pesticides in the PDA medium, measured by percent inhibition/stimulation of fungal growth

\begin{tabular}{llll}
\hline & \multicolumn{2}{l}{ Stimulation/inhibition (\%) } & \\
\cline { 2 - 4 } Pesticide type & A. falavus & A. terres & Average \\
\hline Control & $8.31^{(0.00)}$ & $9.00^{(0.00)}$ & 0.00 \\
Regular M. Galaxy & $1.92^{(76.89)-}$ & $3.46^{(61.55)-}$ & 69.22 \\
Magnetized M. Galaxy & $2.3^{(72.32)-}$ & $3.31^{(63.22)-}$ & 67.77 \\
Regular M. Trayf & $1.63^{(80.38)-}$ & $1.43^{(84.11)-}$ & 82.24 \\
Magnetized M. Trayf & $1.25^{(84.95)-}$ & $0.83^{(90.77)-}$ & 87.86 \\
Average & 62.90 & 59.93 & 61.41 \\
LSD $_{0.0 .05}$ & Pesticide $=$ & Fungi $=$ & Overlab \\
& 1.204 & 0.762 & 1.703 \\
\hline
\end{tabular}

Values are mean of three replicates. Numbers in brackets are representing the percent inhibition/stimulation of each treatment over the control

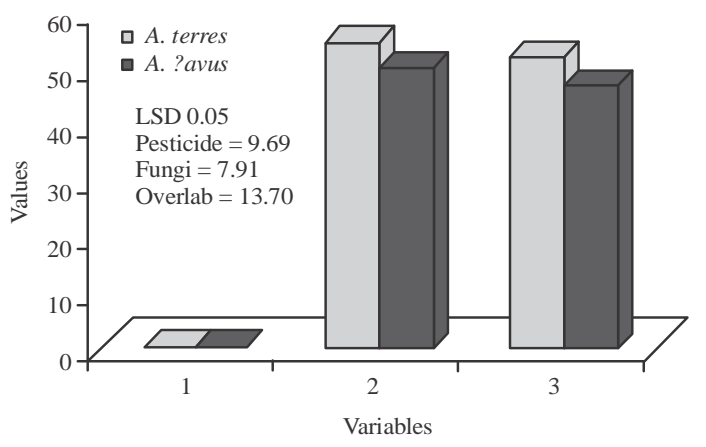

Fig. 3: Effect of exposure to the magnetic field and the presence of fungi, A. flavus and A. terres in degrading chemical compounds of Bayfidan of pesticides in the PDA medium, measured by percent inhibition/stimulation of fungal growth tests on PDB liquid medium

The herbicides Galaxy and Trayf: In case of the herbicides treatments, results (Table 2) showed that there was a significant difference in the percentage of inhibition among fungi tested, that the highest percentage of inhibition was found in the treatment of $A$. flavus and the lowest was recorded in the treatment of $A$. terres, $62.90 \%$ and $59.93 \%$, respectively. The highest percentage of inhibition was always in relation with the herbicides exposed to magnetic field, especially in the Galaxy treatment. The interaction between fungi and exposure to the magnetic field also affected the inhibition rates of fungi growth. The highest percentage of inhibition (90.77\%) occurred in magnetized galaxy treatment interacted with $A$. terres compared to the control treatments of both fungi.

The fungicide Bayfidan: The results shows significant differences in the percentage of inhibition between the two fungi where treated with the fungicide Bayfidan (Fig. 3). A. terres was significantly affected by the fungicide with inhibition rate of $35.4 \%$ compared to A. flavus which was less affected showing inhibition rate of $32 \%$. The inhibition rates were affected by the direct pesticide treatment but not by exposing to the magnetic field. The highest percentage of inhibition (54.33\%) was in the treatment of not magnetized Bayfidan on A. terres compared to the control treatment.

The mechanism of magnetic field in pesticide degradation is not yet known but it is believed that the magnetic field works on T. This is in line with mohassel findings (Mohassel et al., 2009) that the use of magnetized water reduced the surface tension and increased the particle separation of the spray solution and increased the proportional efficiency of targeted control. The process of magnetization of the water through the conditioning of water properties based on magnetic technique lead to polarize water molecules with each other and randomly rearrange water molecules. This process will increase water quality and helps to remove undesirable odors from water such as sulfur and chlorine (Russel, 1987).

Based on calculated fungal fresh and dry biomasses, growth inhibition or stimulation of fungi tested was affected by the pesticide type (Table 3). Generally, the highest rate of growth inhibition was found in the $A$. terres treatments and the lowest rate was observed in the A. flavus treatments. Results (Table 3) shows that the highest rate of inhibition (35.58\%) was found in the A. flavus treatment while A. terres was less affected with an inhibition rate of $33.32 \%$. The rate of inhibition was affected by the pesticide type. Among all pesticides tested, Trayf resulted in the highest inhibition rate $(82.43 \%)$ compared to lowest rate $(0.00)$ resulted from Oberon treatments. Moreover, Oberon resulted in growth stimulation where interacted with both fungi tested on the PDB media.

Although, fungi differed in their growth depend on the pesticide type, this may not give a specific explanation on the level of biological degradation of a pesticide by the fungal isolates. But some other biodegradation indicators such as change in color and odor can be reliable. Generally, the dense growth of a fungus in a toxic environment can indicate the biodegradation ability of that fungus, especially with the increase in hyphal growth which increases the contact area between the fungus cells and the pesticide molecules. This will accelerate the intake process of the pesticide compounds into the fungus body and may also increase the enzymes secretion and consequently increases the level of biodegradation (April et al., 2000; Bennet et al., 2002; Johnsen et al., 2005; Al-Adil, 2006). This is consistent with former studies in which a positive correlation has been found 
J. Eng. Applied Sci., 14 (Special Issue 7): 10131-10136, 2019

Table 3: Effect of exposure to the magnetic field and the presence of fungi, A. flavus and A. terres in degrading chemical compounds of five different types of pesticides in the PDB medium, measured by fresh and dry weight of fungal biomass

\begin{tabular}{|c|c|c|c|c|c|}
\hline \multirow[b]{2}{*}{ Pesticide type } & \multicolumn{2}{|c|}{$\begin{array}{l}\text { Fungal biomass fresh weight }(\mathrm{mL}) \text { from } \\
\text { medium treated with magnetized pesticide }\end{array}$} & \multicolumn{2}{|c|}{$\begin{array}{l}\text { Fungal biomass dry weight }(\mathrm{mL}) \text { from } \\
\text { medium treated with magnetized pesticide }\end{array}$} & \multirow[b]{2}{*}{ Average } \\
\hline & A. falavus & A. terres & A. falavus & A. terres & \\
\hline Control & $0.273^{(0.00)}$ & $0.226^{(0.00)}$ & $0.043^{(0.00)}$ & $0.043^{(0.00)}$ & 0.00 \\
\hline Bulldock & $0.223^{(18.31)-}$ & $0.190^{(15.92)-}$ & $0.033^{(23.25)-}$ & $0.040^{(6.97)-}$ & 14.92 \\
\hline Oberon & $0.406^{(32.75)^{+}}$ & $0.256^{(11.71)^{+}}$ & $0.046^{(6.52)+}$ & $0.043^{(0.00)}$ & 12.74 \\
\hline Galaxy & $0.086^{(68.49)-}$ & $0.056^{(75.22)-}$ & $0.004^{(90.69)-}$ & $0.002^{(95.34)-}$ & 56.74 \\
\hline Trayf & $0.203^{(25.64)-}$ & $0.060^{(73.45)-}$ & $0.023^{(46.51)-}$ & $0.008^{(81.39)-}$ & 82.43 \\
\hline Bayfidan & $0.236^{(13.55)-}$ & $0.190^{(15.92)-}$ & $0.023^{(46.51)-}$ & $0.036^{(16.27)-}$ & 23.06 \\
\hline Average & 25.66 & 32.03 & 35.58 & 33.32 & \\
\hline LSD 0.05 & Pesticide $=6.33$ & & Treatmant $=8.55$ & & Overlab $=15.22$ \\
\hline
\end{tabular}

Values are mean of three replicates. Numbers in brackets are representing the percent inhibition/stimulation of each treatment over the control

between growth increase and biodegradation of hydrocarbon compounds (Rudd et al., 1996; April et al., 2000; Okerentugba and Ezeronye, 2003).

Our results showed a sharp reduction in the biomass final dry weight, especially where fungi were cultured in the pesticide-containing media compared with the control treatment. This decrease in biomass of fungal body may be due to the high content of body fluid in the fungal hyphae resulting from increased surface area of the fungal filaments. This means the increase in contact between the pesticide molecules and fungus cells which accelerates the process of in taking these compounds into the fungus body and storing them in liquid form (April et al., 2000; Bennet et al., 2002; Johnsen et al., 2005).

While the higher values of the dry biomass in the control treatment may be due to the lower amount of nutrients available in the medium which were depleted faster than those of the pesticide-containing medium. This led to fungus shorter life cycle and resulted in spore formation (reproduction units). These spores, generally have higher dry biomass weight that the other body fragments.

\section{CONCLUSION}

However, the fungal growth on the PDB respond differently when a pesticide differed. Magnetized Oberon used in PDB medium resulted in growth stimulation of both fungi, the biomass fresh weight was increased by 11.7 and $32.75 \mathrm{mg}$ for A. flavus and A. terres, respectively. While biomass dry weight was increased $6.52 \mathrm{mg}$ in case of A. flavus on the magnetized Oberon-PDB medium.

\section{REFERENCES}

Al-Adil, K.M., 2006. The Pesticides. Dar Alkutub for Printing and Publishing, Riyadh, Saudi Arabia,.
Al-Malah, N.M. and A. Al-Jubori, 2013. Chemical Pesticides and their Groups, Mechanisms and Metabolisms in Living Organisms and Environment. Dar Al-Yazori for Scientific Publication, Amman, Jordan,.

Al-Mallah, N.M., A.H. Mohammed and A.A. Mustafa, 2012. [The effect of magnetic water and some emulsifiability of physical factor on some pesticides (In Arabic)]. Rafedain J., 40: 254-265.

April, T.M., J.M. Foght and R.S. Currah, 2000. Hydrocarbon degrading filamentous fungi isolated from flare pit soils in Northern and Western Canada. Can. J. Microb., 46: 38-49.

Bennet, J.W., K.G. Wunch and B.D. Faison, 2002. Use of Fungi in Biodegradation. In: Manual of Environmental Microbiology, Hurst, C.J. and R.L. Crawford (Eds.). ASM Press, Washington DC., USA., pp: 959-971.

Carling, D.E. and R.H. Leiner, 1986. Isolation and characterization of Rhizoctonia solani and binucleata $R$. solani-like fungi from aerial stems and subterranean organs of potato plants. Phytopathol., 76: 725-729.

Dewan, M.M., E.L. Ghisalbertib, C. Rowland and K. Sivasithamparam, 1994. Reduction of symptoms of take-all of wheat and rye-grass seedlings by the soil-borne fungus Sordaria fimicola. Appl. Soil Ecol., 1: 45-51.

Gomez, K.A. and A.A. Gomez, 1984. Statistical Procedures for Agricultural Research. 1st Edn., John Wiley and Sons Inc., New York.

Gorski, R. and M. Wachowiak, 2004. Effect of magnetized water on the effectiveness of selected zoocides in the control of red spider mite (Tetranychus urticae Koch) and grain weevil (Sitophilus granaries L.). J. Plant Prot. Res., 44: 13-19.

Harman, G.E., 2000. Myths and dogmas of biocontrol: Changes in perceptions derived from research on Trichoderma harzianum T-22. Plant Dis., 84: 377-393. 
Johnsen, A.R., L.Y. Wick and H. Harms, 2005. Principles of microbial PAH-degradation in soil. Environ. Pollut., 133: 71-84.

Mohassel, M.H.R., A. Aliverdi and R. Ghorbani, 2009. Effects of a magnetic field and adjuvant in the efficacy of cycloxydim and clodinafop propargyl on the control of wild oat (Avena fatua). Weed Biol. Manage., 9: 300-306.

Mondal, G., P. Dureja and B.G. Sen, 2000. Fungal metabolites from Aspergillus niger AN27 related to plant growth promotion. Indian J. Exp. Biol., 38: 84-87.

Okerentugba, P.O. and O.U. Ezeronye, 2003. Petroleum degrading potentials of single and mixed microbial cultures isolated from rivers and refinery effluent in Nigeria. Arf. J. Biotechnol., 2: 288-292.

Rudd, L.E., J.J. Perry, V.S. Houk, R.W. Williams and L.D. Claxton, 1996. Changes in mutagenicity during crude oil degradation by fungi. Biodegrad., 7: 335-343.
Russel, J.A., 1987. Magnetic Water Conditioning Solves Hard Water Problem Without Salt. New Jersey Association of Plumbing-Heating \& Cooling Contractors, Red Bank, New Jersey, USA.,.

Salwan, A.Z.J.H.A., 2015. Effect of some Pesticides and magnetic field and solaration in the soil Microorganism growth. Ph.D Thesis, College of Agriculture, University of Kufa, Kufa, Iraq.

Shaban, A. and N.M. Al-Malah, 1993. Pesticides. Dar Alkutub for Printing and Publishing, Riyadh, Saudi Arabia, Pages: 520.

Sueda, M., A. Katsuki, M. Nonomura, R. Kobayashi and Y. Tanimoto, 2007. Effects of high magnetic field on water surface phenomena. J. Phys. Chem. C., 111: 14389-14393.

Toledo, E.J., T.C. Ramalho and Z.M. Magriotis, 2008. Influence of magnetic field on physical-chemical properties of the liquid water: Insights from experimental and theoretical models. J. Mol. Struct., 888: 409-415. 